\title{
Mid-Upper-Arm Circumference and Arm-to-Height Ratio to Identify Obesity in School-Age Children
}

\author{
Sanguansak Rerksuppaphol, MD and Lakkana Rerksuppaphol, MD
}

\begin{abstract}
Background: In resource-poor settings, anthropometric parameters are evaluated as potential alternatives to the body mass index (BMI) for detecting overweight and obesity in children. To this end, the mid-upperarm circumference (MUAC) and the mid-upper-arm circumference-to-height ratio (AHtR) were evaluated as proxies to BMI in Thai school-age children.
\end{abstract}

Study design: An observational, cross-sectional study was performed on school-aged children.

Participants: Children in grades I through 6 at all public elementary schools in the Ongkharak district, Nakhon Nayok, Thailand during May and June 2013 were included. This is a rural district with low per capita income.

Methods: Weight, height, and MUAC were measured in school-age children and analyzed to identify optimal cut-off values for MUAC and AHtR for detection of overweight and obesity in comparison to BMI. Receiver operating characteristic (ROC) curve analysis determined the validity of MUAC and AHtR use.

Results: Data from 3,618 children, aged 6.0-12.99 years, were analyzed. MUAC correlated with age and height $(P<0.00 \mathrm{I})$, but especially with body weight $(r=0.888$ to 0.914$)$ and BMI $(r=0.859$ to 0.908$)$ in both genders, while AHtR correlated with body weight and BMI $(P<0.00 \mathrm{I})$, but not with age. Cut-off values of MUAC for obesity diagnosis ranged from 18.9 to $25.5 \mathrm{~cm}$ for boys and from 19.8 to $25.4 \mathrm{~cm}$ for girls. Accuracy was excellent for both boys (AUC $=0.952-0.991$ ) and girls (AUC $=0.917-0.990)$. Cut-off of MUAC for overweight diagnosis ranged from 17.2 to $22.4 \mathrm{~cm}$ for boys (AUC $=0.883-0.965$ ) and from 18.0 to $23.2 \mathrm{~cm}$ for girls (AUC $=0.905-0.93 \mathrm{I}$ ). AHtR cut-off values for obesity and overweight diagnosis at 0.16 and 0.145 , respectively, were determined with excellent diagnostic accuracy (AUC ranged from 0.920 to 0.975).

Conclusion: MUAC and AHtR were reliable tools to detect overweight and obesity in Thai school-age children. Cut-off points for MUAC were age and gender specific, while AHtR at 0.16 and 0.145 were the optimal values for both genders, independent of age. These anthropometric measurements showed excellent accuracy in predicting overweight and obesity with high specificity and sensitivity.

Keywords: Anthropometry; Mid-upper-arm circumference; Arm-to-height ratio; Pediatric obesity; Overweight

$\mathrm{T}$ The widespread prevalence of obesity in children and adolescents has become an alarming public health concern in both developed and developing countries. ${ }^{1-3}$

The most common cause of obesity in children is a positive energy balance due to caloric intake in excess of caloric expenditure, combined with a genetic predisposition for weight gain. ${ }^{4}$ Even in developing countries, lack of physical activity and the nutritional transition toward high energy food/junk food have been associated with a rise in obesity prevalence. ${ }^{5,6}$ A previous cross-sectional study of school-age children in Ongkharak, Thailand found a prevalence of $12.8 \%$ for overweight and $9.4 \%$ for obesity, a dramatic increase among Thai children. ${ }^{7}$

Comorbidities that were previously considered "adult" diseases, such as type 2 diabetes mellitus, hypertension,
Corresponding Author: Sanguansak Rerksuppaphol, MD, Department of
Pediatrics, Faculty of Medicine, Srinakharinwirot University, Nakorn Nayok, Thailand
Tel: +66 81723 I766, Fax: +66 37395275, E-mail: sanguansak_r@hotmail.com
Received: March 18, 2017

Ist Revision: July 24, 2017

2nd Revision: September 17, 2017

Accepted: October 2, 2017

doi: $10.3121 / \mathrm{cmr} .2017 .1365$ 
nonalcoholic fatty liver disease, obstructive sleep apnea, and dyslipidemia are now emerging during childhood and adolescence in overweight and obese subjects. ${ }^{4}$ In Thailand, $17.6 \%$ of obese/overweight children presented with metabolic syndrome, ${ }^{8}$ while among obese children, $4.7 \%$ of boys and $3.2 \%$ of girls had hypertension. ${ }^{9}$ The prevalence of dyslipidemia in school-age children was $11.8 \%$, with overweight as the sole risk factor. ${ }^{10}$ Therefore, early identification of children at high risk for obesity should be a pivotal aspect of any obesity prevention initiative. ${ }^{11}$

International guidelines recommend the body mass index (BMI)-for-age as the ideal indicator of obesity for public health surveillance and clinical applications in children and adolescents. $^{12,13}$ However, in resource-poor settings where equipment and training are limited, BMI evaluation may be unreliable and not feasible for use in field studies with many subjects. ${ }^{14}$ Previous studies have indicated that anthropometric measurements of the upper arm were useful parameters for evaluating both under- and over-nutrition in resource-limited countries. ${ }^{14-17}$ Initially, MUAC was developed as a simple and practical proxy for under-nutrition in critical conditions and in emergency settings. ${ }^{18}$ Because of its feasibility and accuracy, MUAC has been considered for early detection of overweight and obesity. In addition, the measurement of MUAC is independent of respiratory movements and postprandial abdominal distension; therefore, it may be a more reliable index than waist circumference. ${ }^{16}$ Craig et al ${ }^{14}$ provided the proof of concept that MUAC could also be used to evaluate over-nutrition. MUAC was accurate for identifying both overweight (defined by BMI) and over-fatness (assessed by bioelectrical impedance) in children aged 5 to 14 years in rural South Africa. Further studies confirmed the usefulness of MUAC for obesity detection. ${ }^{15-17}$ However, cut-off values for MUAC were age and gender specific and varied across ethnic groups. ${ }^{14-17}$ An increasing number of studies have documented that the ratio of mid-upper-arm circumferenceto-height (AHtR) is another accurate tool to screen for childhood obesity. ${ }^{14-17}$ Unlike MUAC, AHtR is not correlated with age and cut-off points can be easily interpreted by both professionals and lay people. ${ }^{16}$

This observational study aimed to calculate optimal cut-off values for MUAC and AHtR for identifying overweight and obese school-aged Thai children.

\section{Materials and Methods}

\section{Study design and population}

An observational, cross-sectional study of school-age children was conducted in the Ongkharak district, Nakhon Nayok province (Thailand) during May and June 2013 to determine the prevalence of obesity in this area. Children in grades 1 through 6 at all public elementary schools in Ongkharak district, located in the province of Nakhon Nayok in central Thailand were eligible to participate in the study. Ongkharak district, with a population of 61,466 (June 2013), has 38 elementary schools. The majority of parents are employed in agriculture and their per capita income was approximately $\$ 2,114$ USD in year 2011 (national per capita income $\$ 4,700$ USD). ${ }^{19}$ Children with significant physical deformities and those with a history of chronic illness, such as chronic respiratory tract infection, chronic liver disease, chronic renal disease, or congenital heart disease were excluded. Children who were unavailable for complete examination on a designated date were also excluded. Written informed consent was obtained. The study was approved by the Ethics Committee of the Faculty of Medicine, Srinakharinwirot University, Thailand.

\section{Parameters}

Anthropometric measurements were performed by staff who were trained in standard measurement protocol as previously described. ${ }^{20}$ Weight was measured in light clothing to the nearest 100 grams using a digital scale (Tanita body composition analyzer, Model BF-680E, Tokyo, Japan). Height was measured to the nearest millimeter using a height rod (Seca, Model 220, Hamburg, Germany). MUAC was evaluated at the midpoint between the olecranon and the acromial process on the upper left-arm using a non-stretch tape with the subject in standing position. BMI was calculated as the ratio of weight $(\mathrm{kg})$ to the square of height $\left(\mathrm{m}^{2}\right)$. In accordance with the age and gender-specific BMI criteria used by the World Health Organization, ${ }^{21}$ obesity was defined as $\mathrm{BMI}>+2$ standard deviation (SD), overweight as BMI > $+1 \mathrm{SD}$, thinness as BMI $<-2 \mathrm{SD}$, and severe thinness as $<-3$ $\mathrm{SD}$. AHtR was calculated as MUAC $(\mathrm{cm})$ to height $(\mathrm{cm})$.

\section{Statistical analysis}

Continuous variables are reported as mean and standard deviation, while categorical variables are reported as frequency and percentage. Pearson's chi-square test and Student's $t$-test were used to compare categorical and continuous variables between genders, respectively. The Pearson correlation coefficient was employed to measure the correlation between 2 variables. Receiver operating characteristic (ROC) analysis was used to determine the performance and cut-off points of MUAC and AHtR for obesity identification. The area under the ROC curve (AUC) described the diagnostic power. Overall diagnostic performance was interpreted as follows: 0.9-1 Excellent; 0.8-0.9 Good; 0.7-0.8 Fair; 0.6-0.7 Poor; and 0.5-0.6 Fail. The optimal cut-off value of MUAC for obesity identification was determined per age and gender; test sensitivity and specificity, and positive (LR+) and negative (LR-) likelihood ratio were also reported. Cut-off values of AHtR were determined per gender group.

Statistical analysis was performed using the SPSS 23.0 software package (SPSS Inc., Chicago, IL, USA). A $P$ value $<0.05$ was considered statistically significant.

\section{Results}

Of the 4,219 school-age children eligible for consideration in the study, 228 children were excluded for the following 
Table 1. Demographic characteristics

\begin{tabular}{llll}
\hline Variables & Boys $(\mathbf{n}=\mathbf{1 , 8 3 0})$ & Girls $(\mathbf{n}=\mathbf{1 , 7 8 8})$ & $\boldsymbol{P}$ \\
\hline Age $(\mathrm{yr})$ & $9.8 \pm 1.7$ & $9.8 \pm 1.7$ & 0.533 \\
Weight $(\mathrm{kg})$ & $33.1 \pm 12.6$ & $33.3 \pm 12.4$ & 0.590 \\
Height $(\mathrm{cm})$ & $133.5 \pm 11.8$ & $134.6 \pm 12.5$ & 0.010 \\
Body mass index $\left(\mathrm{kg} / \mathrm{m}^{2}\right)$ & $18.06 \pm 4.46$ & $17.91 \pm 4.45$ & 0.309 \\
Mid-upper arm circumference $(\mathrm{cm})$ & $19.5 \pm 4.0$ & $19.3 \pm 3.7$ & 0.280 \\
Arm-to-height ratio & $0.145 \pm 0.024$ & $0.143 \pm 0.022$ & 0.010 \\
Nutritional status, no. $(\%)$ & & & $<0.001$ \\
$\quad$ Obesity & $345(18.9)$ & $234(13.1)$ & $251(14.0)$ \\
$\quad$ Overweight & $242(13.2)$ & $1216(68.0)$ & $68(3.8)$ \\
$\quad$ Normal & $1138(62.2)$ & $19(1.1)$ & \\
$\quad$ Thinness & $82(4.5)$ & & \\
\hline
\end{tabular}

reasons: lack of informed consent (115), being absent on the scheduled day (93), incomplete measurement data (10), and moving away from the school (10). Of the 3,991 children enrolled in the study, MUAC and AHtR measurements were available for 3,873 children. Data from children younger than 7 years old (117 boys and 138 girls) were excluded from the analysis, due to the sample size being too small to obtain a reliable statistical power. Therefore, data from 3,618 children were considered in the final analysis.

The mean age of children included in the analysis was 9.8 years (7.0-12.9 years), the mean weight was $33.2 \mathrm{~kg}$ (13.4 $116.7 \mathrm{~kg})$ and the BMI was $17.98 \mathrm{~kg} / \mathrm{m}^{2}\left(9.85-57.21 \mathrm{~kg} / \mathrm{m}^{2}\right)$. Overall, $50.6 \%$ of children included in the study were male.

Demographic characteristics are summarized in Table 1. Age, body weight, BMI, and MUAC were similar among boys and girls; boys were shorter $(P=0.010)$ and had higher AHtR $(P$ $=0.010)$. The overall prevalence of obesity was $16.0 \%$, significantly higher in boys than in girls $(18.9 \%$ and $13.1 \%$; $P<0.001)$. The prevalence of overweight, thinness and severe thinness were $13.6 \%, 4.1 \%$ and $1.2 \%$, respectively, without significant differences between genders.

MUAC correlated with all parameters $(P<0.001)$, especially with body weight ( $r=0.859$ to 0.914$)$ and BMI ( $r=0.888$ to $0.908)$ in both genders. AHtR correlated with body weight and BMI $(P<0.001)$, but not with age (Table 2$)$.

Cut-off values and ROC curves of MUAC for obesity and overweight diagnosis per gender and age are presented in Table 3. Cut-off values of MUAC for obesity diagnosis ranged from $18.9 \mathrm{~cm}$ to $25.5 \mathrm{~cm}$ for boys and from $19.8 \mathrm{~cm}$ to $25.4 \mathrm{~cm}$ for girls. The AUC was excellent for both boys (0.952-0.991) and girls (0.917-0.990). Sensitivity and specificity were high for all of age and gender-specific cut-off values (sensitivity $81.8 \%$ - 95.7\%; specificity 90.9\% 98.3\%); LR+ ranged from 9.81 to 55.82 and LR- ranged from 0.04 to 0.19 . Cut-off values of MUAC for overweight ranged from $17.2 \mathrm{~cm}$ to $22.4 \mathrm{~cm}$ for boys and from $18.0 \mathrm{~cm}$ to $23.2 \mathrm{~cm}$ for girls. The AUC was good to excellent for the diagnosis of overweight in both genders $(0.883-0.965$ in boys and $0.905-0.931$ in girls).

Similarly, the accuracy of AHtR for diagnosis of obesity was excellent (AUC $=0.975$ in boys and AUC $=0.944$ in girls). AHtR cut-off value was 0.16 and it provided high sensitivity and specificity in predicting obesity (sensitivity $93.9 \%$ in boys and $87.6 \%$ in girls; specificity $92.9 \%$ in boys and $91.8 \%$ in girls); $\mathrm{LH}+$ ranged from 10.68 to 13.23 and LR- from 0.07 to 0.14 . At the cut-off value of 0.145 , AHtR provided relatively high sensitivity and specificity in predicting overweight in both genders. The calculated optimal cut-off values of AHtR are detailed in Table 4.

\section{Discussion}

This observational study demonstrated that MUAC and AHtR were reliable indices for obesity and overweight identification in Thai school-age children, with high sensitivity and specificity.

Evaluation of overweight and obesity is usually based on the definition of BMI..$^{12,13,21}$ However, BMI offers little indication of body fat distribution..$^{22}$ Body composition is an important indicator of health status in children and adolescents because maintaining a healthy body composition prevents the onset of obesity that is associated with the risk of cardiovascular diseases, diabetes and stroke. ${ }^{22}$ Waist circumference and upper arm anthropometric measures may contribute to distinguishing a central distribution of body fat, particularly an excess intra-abdominal accumulation of fat rather than a more peripheral distribution. In addition, these parameters show a good level of correlation with corporal mass and may be useful to control body growth during childhood and adolescence. ${ }^{23}$ As expected, MUAC increases with age and varies between genders. Craig et $\mathrm{al}^{14}$ investigated the 
Table 2 Correlation between mid-upper-arm circumference, arm-to-height ratio and age, body weight, height and body mass index by gender.

\begin{tabular}{|c|c|c|c|c|c|c|c|c|}
\hline & \multicolumn{4}{|c|}{ MUAC } & \multicolumn{4}{|c|}{ AHtR } \\
\hline & \multicolumn{2}{|c|}{ Boys } & \multicolumn{2}{|c|}{ Girls } & \multicolumn{2}{|c|}{ Boys } & \multicolumn{2}{|c|}{ Girls } \\
\hline & $r$ & $P$ & $r$ & $P$ & $r$ & $P$ & $r$ & $P$ \\
\hline Age & 0.347 & $<0.001$ & 0.398 & $<0.001$ & 0.003 & 0.889 & -0.008 & 0.736 \\
\hline Weight & 0.914 & $<0.001$ & 0.888 & $<0.001$ & 0.702 & $<0.001$ & 0.621 & $<0.001$ \\
\hline Height & 0.603 & $<0.001$ & 0.583 & $<0.001$ & 0.212 & $<0.001$ & 0.114 & $<0.001$ \\
\hline BMI & 0.908 & $<0.001$ & 0.859 & $<0.001$ & 0.879 & $<0.001$ & 0.826 & $<0.001$ \\
\hline
\end{tabular}

MUAC = mid-upper-arm circumference; $\mathrm{AHtR}=$ arm-to-height ratio

usefulness of MUAC for describing obesity prevalence in children from rural South Africa. As a proof of concept study, only two age groups were considered (5-9 years and 10-14 years), with poor accuracy of MUAC cut-off points for 5-9 year old boys..$^{14}$ When calculated for each age, cut-off values for MUAC showed a robust diagnostic performance and a powerful ability to identify Han children with or without elevated BMI. ${ }^{16}$ Mazicioglu et $\mathrm{a}^{17}$ further specified the ages for clinically significant cut-offs: $9-14$ and $16-17$ for boys and $15-16$ for girls. Although these data seem promising, it should be noted that systematic monitoring of MUAC is not commonly performed in pediatric patients in many countries and internationally accepted cut-off values have not yet been established.

In this study, AHtR was also considered as an anthropometric parameter to identify obese and overweight Thai children. AHtR has an important clinical and practical advantage compared to other upper arm anthropometric measurements: it is not correlated with age, likely because AHtR is already adjusted by height, which is strongly correlated with age. ${ }^{16}$ For the pediatric population, growth is a very important factor for body composition change; therefore, age and height should always be considered. To our knowledge, only one study investigated the potential usefulness of AHtR to screen children for overweight and obesity. Lu et $\mathrm{al}^{16}$ identified an AHtR cut-off point at 0.15 in Han children; this value had excellent accuracy in terms of both specificity and sensitivity. In our study, the optimal cut-off for obesity diagnosis for school-age Thai children was 0.16 . The minimal difference among cut-off values may be explained by the different obesity definitions used in the studies rather than by the ethnicity. However, in both cases, the accuracy of AHtR to detect obesity was excellent for both genders, thus suggesting that AHtR is a feasible tool to screen children for obesity in community and non-professional settings. In our study, using the cut-off AHtR at 0.145 for overweight diagnosis gave excellent accuracy. Early detection of overweight status may help prevent later obesity. To our knowledge, this is the first report using AHtR for the diagnosis of overweight.

This study had some limitations. We did not directly measure body fat, but we considered BMI as a standard criterion to classify overweight and obesity in the study. BMI is widely considered a gold standard for detecting obesity in epidemiological studies; however, in Thailand, the use of BMI-for-age is not recommended in the public health system, due to lack of standard scale in Thai children. AHtR is not dependent on age and may prove valuable in this respect. Furthermore, tape measures and height rods are easier to transport to non-clinical or resource-poor settings, making the use of MUAC and AHtR more ubiquitous and practical than BMI in medical settings. In addition, MUAC and AHtR can both be calculated by laypeople at home, enabling them to remain actively involved in their own health maintenance. The power of the two measurements validated in this study is inherent in their ability to be used in any setting with little education. Almost anyone can measure a height and an arm circumference. Thereafter, publicly-available tables with cutoffs can facilitate the diagnosis of obesity and overweight in children. To our knowledge, this study provides the first determination of MUAC and AHtR cut-offs for obesity and overweight detection in a population of school-age Thai children. Further studies are required to validate our findings and to apply them to a larger portion of the pediatric population in Thailand.

\section{Conclusion}

MUAC and AHtR were reliable tools to detect obesity and overweight in Thai school-age children. Cut-off points for MUAC were age- and gender-specific, while AHtR at 0.16 (for obesity) and at 0.145 (for overweight) were the optimal cut-off values for both genders, independent of age. These anthropometric measurements showed excellent accuracy in predicting obesity with high specificity and sensitivity. Further studies will support the usefulness of MUAC and AHtR to determine the prevalence of obesity in different age groups (e.g., infants and adolescents) or to assess the prevalence of dyslipidemia or type-2 diabetes in young populations.

\section{Acknowledgements}

We thank Elisa Sala, PhD, Medical Writer, for her medical editorial assistance with our report. 
虫

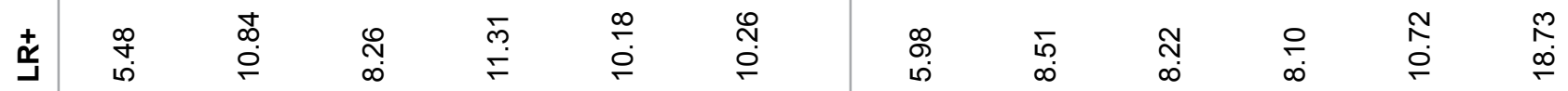

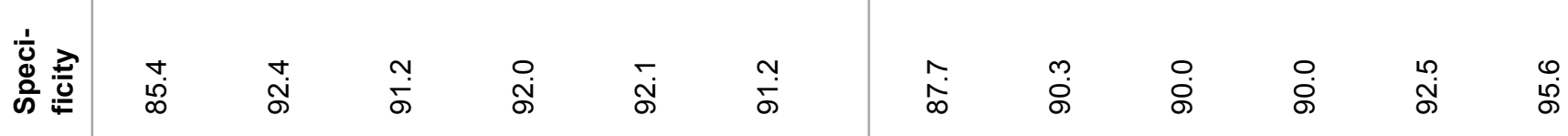
ஸे

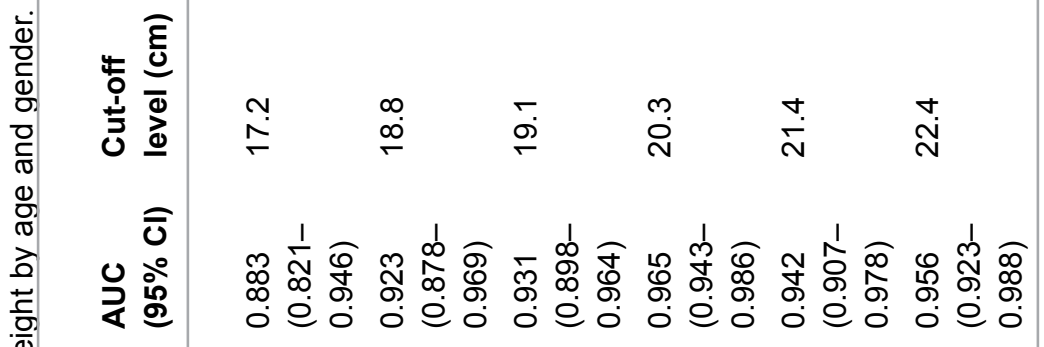

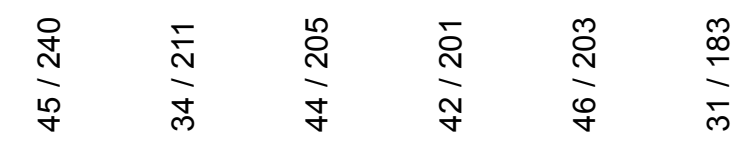

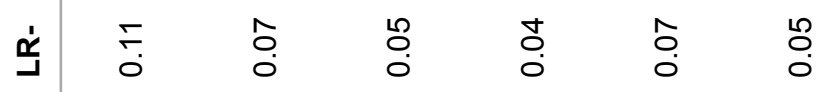

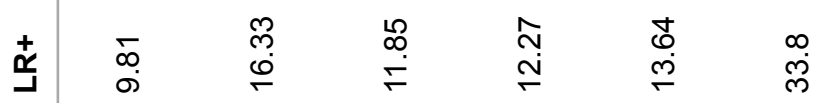

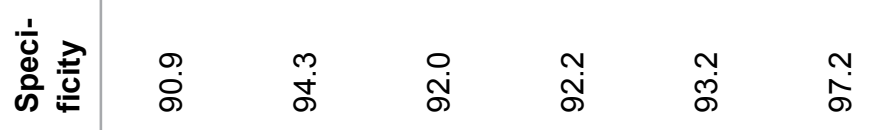

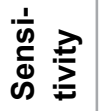

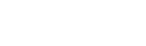

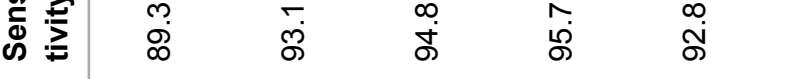

告

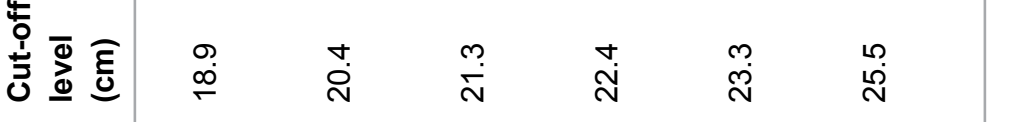

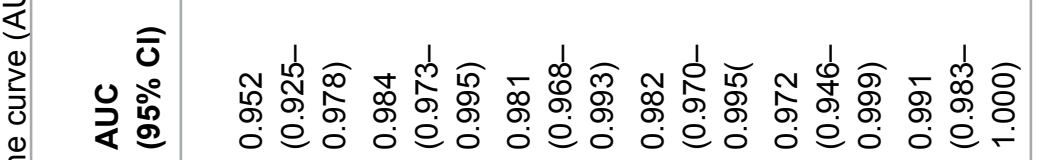

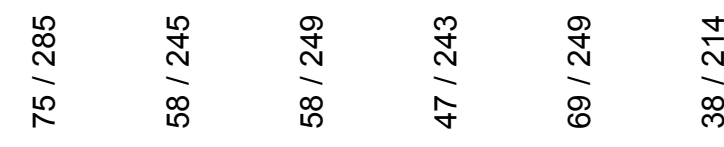

$\underset{\substack{\infty \\ \infty}}{\stackrel{ \pm}{N}}$

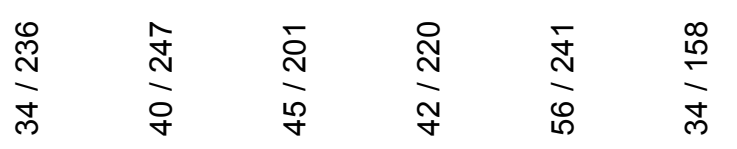

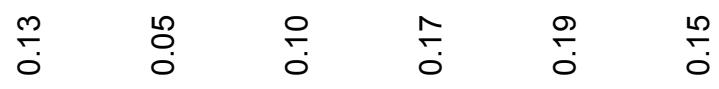

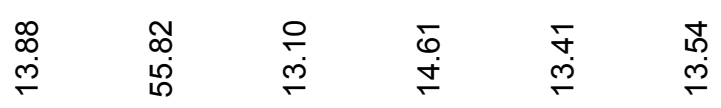

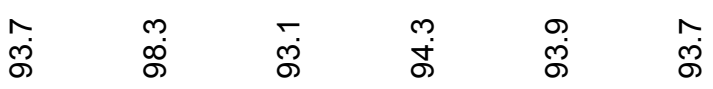

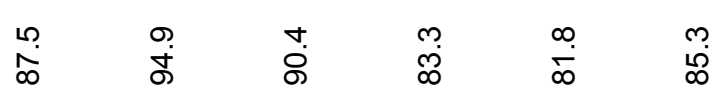

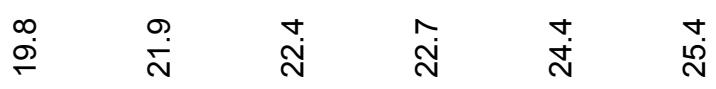

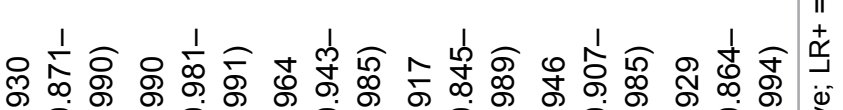

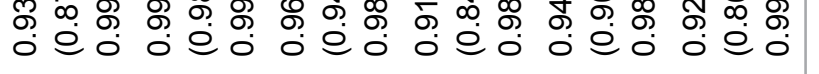

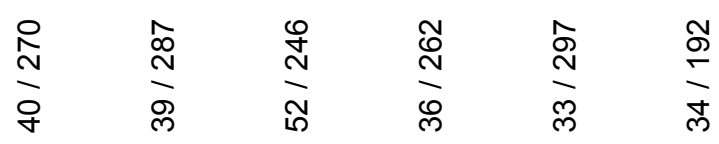

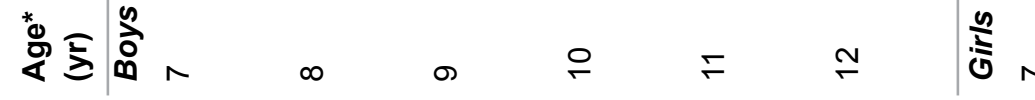


Table 4. Area under the curve (AUC) of arm-to-height ratio for the diagnosis of obesity and overweight by gender.

\begin{tabular}{|c|c|c|c|c|c|c|c|c|}
\hline Gender & $\begin{array}{l}\text { Nutritional } \\
\text { status }\end{array}$ & $\mathbf{n}$ & AUC $(95 \% \mathrm{Cl})$ & $\begin{array}{l}\text { Cut-off } \\
\text { level }\end{array}$ & Sensitivity & Specificity & LR+ & LR- \\
\hline \multirow[t]{2}{*}{ Boys } & Obesity & 345 & $\begin{array}{l}0.975(0.967- \\
0.983)\end{array}$ & 0.16 & 93.9 & 92.9 & 13.23 & 0.07 \\
\hline & Overweight & 242 & $\begin{array}{l}0.929(0.912- \\
0.946)\end{array}$ & 0.145 & 87.6 & 86.6 & 6.54 & 0.14 \\
\hline \multirow[t]{2}{*}{ Girls } & Obesity & 234 & $\begin{array}{l}0.944(0.925- \\
0.964)\end{array}$ & 0.16 & 87.6 & 91.8 & 10.68 & 0.14 \\
\hline & Overweight & 251 & $\begin{array}{l}0.920(0.902- \\
0.939)\end{array}$ & 0.145 & 86.1 & 83.1 & 5.09 & 0.17 \\
\hline
\end{tabular}

AUC, Area under the curve; LR+, positive likelihood ratio; LR-, negative likelihood ratio

\section{References}

1. Choukem SP, Kamdeu-Chedeu J, Leary SD, et al. Overweight and obesity in children aged 3-13 years in urban Cameroon: a cross-sectional study of prevalence and association with socio-economic status. BMC Obesity. 2017;4(1):7.

2. Zhang Y, Wang Z, Zhao J, Chu Z. The current prevalence and regional disparities in general and central obesity among children and adolescents in Shandong, China. Int J Cardiol. 2017;227:89-93.

3. Ha KH, Kim DJ. Epidemiology of childhood obesity in Korea [Published correction appears in Endocrinol Metab (Seoul). 2017;32(1):144]. Endocrinol Metab (Seoul). 2016;31(4):510518.

4. Kumar S, Kelly AS. Review of childhood obesity: from epidemiology, etiology, and comorbidities to clinical assessment and treatment. Mayo Clin Proc. 2017;92(2):251265.

5. Mistry SK, Puthussery S. Risk factors of overweight and obesity in childhood and adolescence in South Asian countries: a systematic review of the evidence. Public Health. 2015;129(3):200-209.

6. Poskitt EME. Childhood obesity in low- and middle-income countries. Paediatrics and International Child Health. 2014;34(4):239-249.

7. Rerksuppaphol S, Rerksuppaphol L. Prevalence of overweight and obesity among school children in suburb Thailand defined by the International Obesity Task Force Standard. [corrected]. J Med Assoc Thai. 2010;93(Suppl 2):S27-S31.

8. Rerksuppaphol L, Rerksuppaphol S. Prevalence of Metabolic Syndrome in Thai Children: A Cross-sectional Study. J Clin Diagn Res. 2014;8(4):PC04-PC07.

9. Sukhonthachit P, Aekplakorn W, Hudthagosol C, Sirikulchayanonta $\mathrm{C}$. The association between obesity and blood pressure in Thai public school children. BMC Public Health. 2014;14(1):729.

10. Rerksuppaphol S, Rerksuppaphol L. Prevalence of dyslipidemia in Thai schoolchildren. J Med Assoc Thai. 2011;94(6):710715.

11. Manios Y, Vlachopapadopoulou E, Moschonis G, et al. Utility and applicability of the Childhood Obesity Risk Evaluation (CORE)-index in predicting obesity in childhood and adolescence in Greece from early life: the National Action Plan for Public Health. Eur J Pediatr. 2016;175(12):19891996.

12. Cole TJ, Bellizzi MC, Flegal KM, Dietz WH. Establishing a standard definition for child overweight and obesity worldwide: international survey. BMJ. 2000;320(7244):12401243.

13. Ahmad QI, Ahmad CB, Ahmad SM. Childhood obesity. Indian J Endocrinol Metab. 2010;14(1):19-25.
14. Craig E, Bland R, Ndirangu J, Reilly JJ. Use of mid-upper arm circumference for determining overweight and overfatness in children and adolescents. Arch Dis Child. 2014;99(8):763766.

15. Chaput JP, Katzmarzyk PT, Barnes JD, et al; ISCOLE Research Group. Mid-upper arm circumference as a screening tool for identifying children with obesity: a 12-country study. Pediatr Obes. 2017;12(6):436-445.

16. Lu Q, Wang R, Lou DH, Ma CM, Liu XL, Yin FZ. Mid-upperarm circumference and arm-to-height ratio in evaluation of overweight and obesity in Han children. Pediatr Neonatol. 2014;55(1):14-19.

17. Mazıcıoğlu M, Hatipoğlu N, Öztürk A, Çiçek B, Üstünbaş HB, Kurtoğlu S. Waist circumference and mid-upper arm circumference in evaluation of obesity in children aged between 6 and 17 years. J Clin Res Pediatr Endocrinol. 2010;2(4):144-150.

18. Ralston ME, Myatt MA. Weight Estimation Tool for Children Aged 6 to 59 Months in Limited-Resource Settings. PLoS ONE. 2016;11(8):e0159260.

19. Ministry of Labour of Thailand. Economic performance and social conditions of Nakorn Nayok province. [cited 2017 July 20]. Available from: http://nakhonnayok.mol.go.th/system/ files/bththii_1_ phaawaesrsthkicchaelasangkhm_8.pdf.

20. Rerksuppaphol S, Rerksuppaphol L. Association of obesity with the prevalence of hypertension in school children from central Thailand. J Res Health Sci. 2015;15(1):17-21.

21. Lima AAM, Soares AM, Lima NL, et al. Effects of vitamin A supplementation on intestinal barrier function, growth, total parasitic, and specific Giardia spp infections in Brazilian children: a prospective randomized, double-blind, placebocontrolled trial. J Pediatr Gastroenterol Nutr. 2010;50(3):309315.

22. McCarthy HD. Measuring growth and obesity across childhood and adolescence. Proc Nutr Soc. 2014;73(02):210-217.

23. Jelliffe EF, Jelliffe DB. Anthropometry in action. I. Dental second year malnutrition. (Practical age-grouping in young children in areas without birth verification). J Trop Pediatr (1967). 1968;14(2):71-74.

\section{Author Affiliations}

Sanguansak Rerksuppaphol, MD*; and Lakkana Rerksuppaphol, MD广

*Department of Pediatrics, Faculty of Medicine, Srinakharinwirot University, Nakorn Nayok, Thailand, E-mail:sanguansak_r@hotmail.com.

$\dagger$ Department of Preventive Medicine, Faculty of Medicine, Srinakharinwirot University, Nakorn Nayok, Thailand, E-mail:lakkana_r@hotmail.com. 